\title{
Profile of pembrolizumab in the treatment of patients with unresectable or metastatic urothelial carcinoma
}

This article was published in the following Dove Press journal: Cancer Management and Research

\section{Junichi Inokuchi \\ Masatoshi Eto}

Department of Urology, Graduate School of Medical Sciences, Kyushu University, Fukuoka, Japan
Correspondence: Junichi Inokuchi Department of Urology, Graduate School of Medical Sciences, Kyushu University, 3-I-I Maidashi, Higashi-ku, Fukuoka 8I2-8582, Japan

Tel +8I 926425603

Fax +81926425618

Email junichi@uro.med.kyushu-u.ac.jp
Abstract: The prognosis of patients with unresectable or metastatic urothelial carcinoma (UC) is poor. Platinum-based chemotherapy has been the standard first-line treatment in these patients for the past decade; however, the 5-year overall survival (OS) rate is only 13-22\%. Recent advances in cancer immunology research have highlighted the pivotal role of the immune system in cancer development and progression, and new immune checkpoint inhibitors (ICIs) have demonstrated efficacy in a large variety of tumors including UC. Currently, five ICIs, including two anti-PD-1 antibodies (pembrolizumab and nivolumab) and three anti-PD-L1 antibodies (atezolizumab, avelumab, and durvalumab), have been granted approval by the US Food and Drug Administration (FDA) for patients with unresectable or metastatic UC who recurred or progressed after platinum-based chemotherapy. Among these agents, only pembrolizumab is supported by strong evidence from a large randomized Phase III trial (KEYNOTE-045). This trial demonstrated statistically significant improvements in OS for patients assigned to the pembrolizumab arm compared with the chemotherapy arm, both in the total population (HR $0.73 ; P=0.002$ ) and in the population with high PD-L1 expression (HR 0.57; $P=0.005$ ). For patients with cisplatin-ineligible UC, pembrolizumab and atezolizumab were approved based on Phase II studies, with limitations on the use of these agents in patients with high tumor PD-L1 expression later imposed by the FDA. In conclusion, pembrolizumab may be a potential first-choice second-line therapy for unresectable or metastatic UC patients following platinum-based chemotherapy. Several Phase III trials are ongoing to evaluate the efficacy and toxicity of combination therapies of ICIs with chemotherapy, and ICIs with other ICIs with or without chemotherapy as firstline therapy. The results of these trials might redirect treatment strategies for patients with unresectable or metastatic UC.

Keywords: urothelial carcinoma, pembrolizumab, PD-1, immune checkpoint inhibitor, PD-L1

\section{Introduction}

Urothelial carcinoma (UC) can arise from the urothelium of the entire urinary tract, including upper urinary tract (renal pelvis and ureter) and urethra, but most commonly from bladder. Bladder cancer (BC) is the seventh most commonly diagnosed cancer in the male population worldwide, and the eleventh in both sexes. ${ }^{1}$ Approximately $75 \%$ of patients are diagnosed with non-muscle invasive cancer, and are managed with transurethral resection and intravesical instillation of Bacillus Calmette-Guerin (BCG) or chemotherapeutic agents. ${ }^{2}$ These patients show relatively favorable prognosis, while patients with muscle-invasive $\mathrm{BC}$ showed 
poor prognosis and the 5-year overall survival (OS) rate of less than $50 \%{ }^{3}$ Ten to fifteen percent of patients show metastatic disease at initial diagnosis, and the prognosis of these patients is poor. ${ }^{4}$

Platinum-based chemotherapy has been the standard first-line treatment in patients with unresectable or metastatic UC for the past decade. The MVAC (methotrexate, vinblastine, doxorubicine, and cisplatin) regimen was introduced with good objective response rate of $69 \%{ }^{5}$ Recently, a regimen of dose-dense MVAC was introduced, which showed more favorable toxicity profile compared with the original MVAC regimen, higher complete response (CR) rate ( $21 \%$ vs. $9 \%, P=0.009)$, higher overall response rate (ORR) (64\% vs. $50 \%, P=0.06)$, and comparable median OS (15.1 vs. 14.9 months) ${ }^{6,7}$ The other commonly used regimen is GC (gemcitabine and cisplatin), which showed comparable OS to the original MVAC (median 14.0 vs. 15.2 months) (hazard ratio [HR], 1.09; $P=0.66$ ), and fewer toxicities. ${ }^{8}$ Despite these improvements and modifications, the median OS in patients who received cisplatin-based chemotherapy was approximately 15 months, and the 5-year OS rate was $13-22 \%{ }^{4,7,8}$

Recent advances in the understanding of immunological mechanisms against malignant neoplasms have initiated a revolution in the treatment of various solid malignancies including UC. Immune checkpoint inhibitors (ICIs) have been the leading drugs in this field, and upregulate anti-tumor activity by inhibiting the immune escape mechanism in tumor cells and immune cells. ${ }^{9}$ This review summarizes critical data from recent clinical trials of ICIs for unresectable or metastatic UC, and describes pembrolizumab, currently the only agent with strong evidence.

\section{Immune check point inhibitors (ICls)}

Recent advances in the cancer immunology field have highlighted the pivotal role of the immune system in cancer development and progression. ${ }^{10}$ Under physiological conditions, the immune system eliminates tumor cells based on its recognition of tumor-specific antigens. Cytotoxic T cells and natural killer (NK) cells are the main effectors that attack tumor cells, either directly or indirectly, and activation of these cells is tightly regulated by multiple co-stimulatory and co-inhibitory receptor signals, so-called immune checkpoints, and inflammatory cytokines. However, tumor cells can evade the host anti-tumor response through activation of co-inhibitory receptors, such as programmed cell death 1
(PD-1) and cytotoxic T lymphocyte-associated protein 4 (CTLA-4). PD-1 has two main ligands, programmed death ligand 1 and 2 (PD-L1/PD-L2) which are expressed on antigen-presenting cells (APCs) and cancer cells, and interactions with PD-1 and PD-L1/PD-L2 inhibit T cell function. Therefore, targeting these immune checkpoints is expected to lead to enhanced anti-tumor responses in a large variety of tumors including UC (Figure 1).

Anti-CTLA-4 antibodies have been reported to have excellent therapeutic effects in advanced melanoma patients. ${ }^{11}$ Anti-PD-1 or anti-PD-L1 antibodies, inhibitors of the PD-1/ PD-L1 pathway, were subsequently developed and used to treat patients with kidney cancer, non-small cell lung cancer, and malignant melanoma, with favorable therapeutic effects. ${ }^{9}$

\section{ICls for post-platinum-based chemotherapy setting}

Since May 2006, five ICIs, including two anti-PD-1 antibodies (pembrolizumab and nivolumab) and three anti-PD -L1 antibodies (atezolizumab, avelumab, and durvalumab), have been granted approval by the US Food and Drug Administration (FDA) for patients with unresectable or metastatic US who exhibited disease progression during or following platinum-based chemotherapy (Table 1). Among these agents, only pembrolizumab is supported by strong evidence from a large randomized Phase III trial (KEYNOTE-045); therefore, the FDA granted regular approval for pembrolizumab in May 2017, while the others have received accelerated approval.

\section{Pembrolizumab}

Pembrolizumab, a humanized monoclonal $\mathrm{IgG} 4 \kappa$ isotype antibody against PD-1, showed anti-tumor activity in patients with unresectable or metastatic $\mathrm{UC}$ in the Phase $\mathrm{Ib}$ KEYNOTE-012 study. ${ }^{12}$ In this study, 33 patients with positive PD-L1 expression who were diagnosed with unresectable or metastatic UC were enrolled. The ORR at the median follow-up of 13 months was $26 \%$ with a CR rate of $11 \%$, and the safety profile was acceptable. This study supported the rationale for a Phase II study (KEYNOTE-052) and a Phase III trial (KEYNOTE-045). ${ }^{13,14}$

KEYNOTE-045 was a Phase III open-label randomized trial that compared pembrolizumab with the investigator's choice of chemotherapy, including paclitaxel, docetaxel, and vinflunine, in patients with unresectable or metastatic UC that recurred or progressed after platinum-based chemotherapy. ${ }^{13}$ A total of 542 patients were randomly assigned in a 1:1 ratio to 


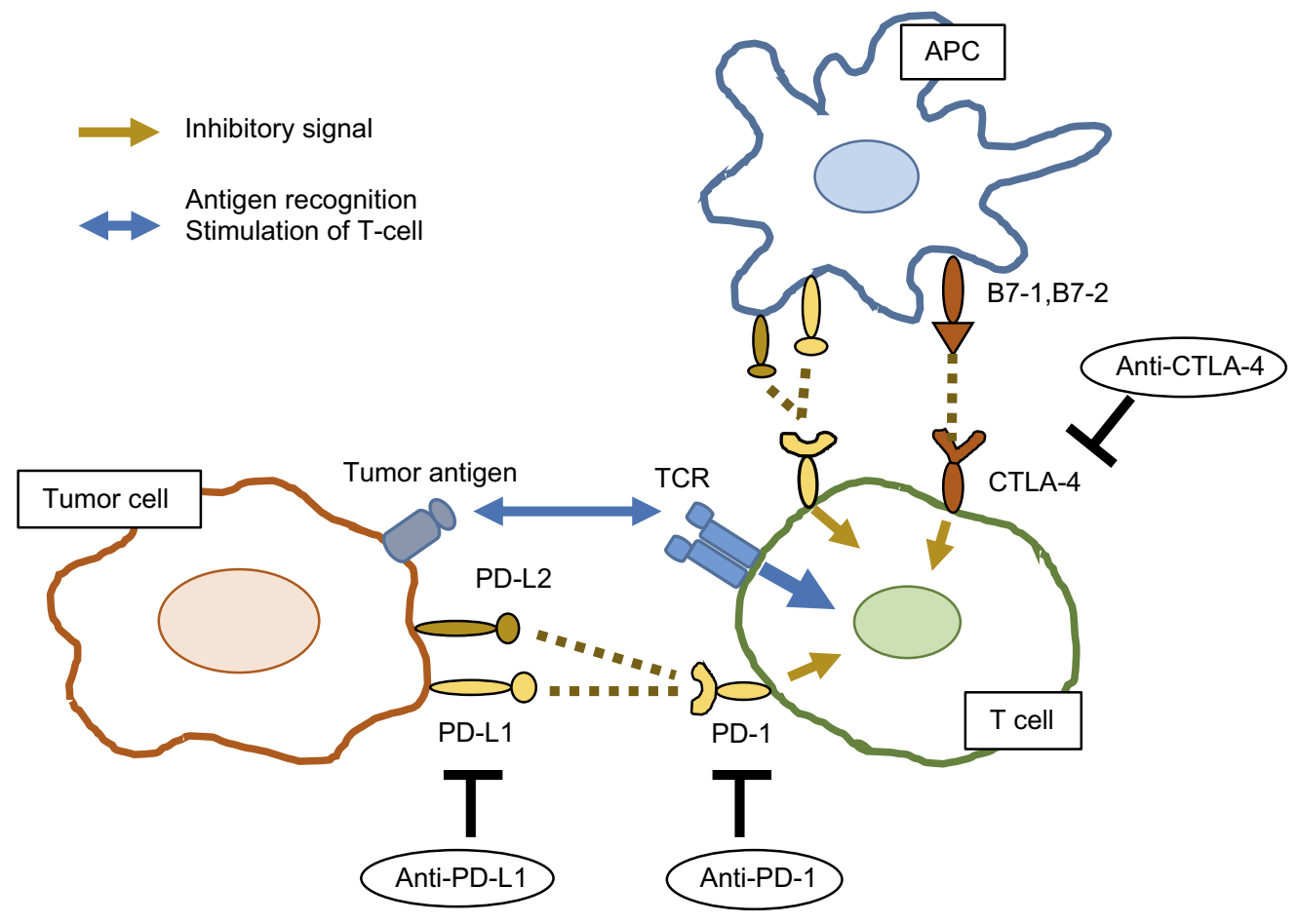

Figure I Mechanisms of action of ICls.

Abbreviations: APC, antigen-presenting cell; TCR, T cell receptor; PD-I, programmed cell death I; PD-L, programmed death ligand; CTLA-4, cytotoxic T lymphocyteassociated protein 4 .

Table I Immune checkpoint inhibitors (ICls) for patients with unresectable or metastatic urothelial carcinoma (UC)

\begin{tabular}{|c|c|c|c|c|c|}
\hline & Target & Type & FDA approval & $\begin{array}{l}\text { Trial phase } \\
\text { based on } \\
\text { approval }\end{array}$ & Assay for PD-LI expression \\
\hline Pembrolizumab & PD-I & $\lg G 4$ & $\begin{array}{l}\text { Post-platinum-based chemotherapy* } \\
\text { First-line, cisplatin-ineligible }\end{array}$ & $\begin{array}{l}\text { Phase III } \\
\text { Phase II }\end{array}$ & Dako PD-LI IHC 22 C3 pharmDx assay \\
\hline Nivolumab & PD-I & $\lg G 4$ & Post-platinum-based chemotherapy & Phase II & Dako PD-LI IHC 28-8 pharmDx assay \\
\hline Atezolizmab & PD-LI & $\lg G \mid$ & $\begin{array}{l}\text { Post-platinum-based chemotherapy } \\
\text { First-line, cisplatin-ineligible }\end{array}$ & $\begin{array}{l}\text { Phase II } \\
\text { Phase II }\end{array}$ & VENTANA PD-LI (SPI42) assay \\
\hline Durvalumab & PD-LI & $\operatorname{lgG} \mid$ & Post-platinum-based chemotherapy & Phase I/II & VENTANA PD-LI (SP263) assay \\
\hline Avelumab & PD-LI & $\lg G \mid$ & Post-platinum-based chemotherapy & Phase lb & Dako PD-LI IHC73-I0 pharmDx assay \\
\hline
\end{tabular}

Note: *Regular approval.

receive either pembrolizumab $200 \mathrm{mg}$ every 3 weeks $(\mathrm{n}=270)$ or the investigator's choice of a chemotherapy regimen (paclitaxel $[\mathrm{n}=84]$, docetaxel $[\mathrm{n}=84]$, or vinflunine $[\mathrm{n}=87])$ every 3 weeks ( $\mathrm{n}=272$ ). The co-primary endpoints in this trial were OS and progression-free survival (PFS), which were assessed in the total population and in the population with high tumor PDL1 expression. In this trial, PD-L1 expression was assessed using the PD-L1 IHC 22C3 pharmDx assay (Dako North America, Inc., Carpinteria, CA), and high expression was defined as the PD-L1 combined positive score (CPS, the percentage of PD-L1-expressing tumor and infiltrating immune cells relative to the total number of tumor cells) of $10 \%$ or more.
This trial demonstrated statistically significant improvements in OS for patients assigned to the pembrolizumab arm compared with the chemotherapy arm, both in the total population and in the population with high PD-L1 expression. The median OS in the total population was 10.3 and 7.4 months in the pembrolizumab and chemotherapy arms, respectively (HR $0.73 ; 95 \%$ CI: $0.59-0.91 ; P=0.002$ ). The median OS in the population with high PD-L1 expression was 8.0 and 5.2 months, respectively (HR $0.57 ; 95 \% \mathrm{CI}$ : $0.37-0.88 ; P=0.005)$. However, no statistically significant difference in PFS between the two arms was observed. The median PFS in the total population was 2.1 and 3.3 months in the pembrolizumab and chemotherapy arms, respectively 
(HR 0.98; 95\% CI: $0.81-1.19 ; P=0.42$ ). The PFS in the population with high PD-L1 expression showed a similar tendency (HR 0.89; 95\% CI: $0.61-1.28 ; P=0.24$ ). ORR was $21 \%$ for pembrolizumab and $11 \%$ for chemotherapy $(P=0.001)$ in the total population. The duration of response was longer in patients who responded to pembrolizumab than in those with a response to chemotherapy. The estimated percentage of patients with a response duration of at least 12 months was $68 \%$ and $35 \%$ in the pembrolizumab and chemotherapy arms, respectively. Adverse events (AEs) were reported at a lower rate in the pembrolizumab arm than in the chemotherapy arm (any grade: 61\% vs. 90\%; grade 3 , 4 , or 5 : $15 \%$ vs. $49 \%$ ). Based on the results of this trial, pembrolizumab was granted regular approval by the FDA for patients with unresectable or metastatic UC, regardless of PD-L1 status.

\section{Other ICls (atezolizumab, nivolumab, avelumab, and durvalumab)}

Atezolizumab, an anti-PD-L1 antibody, was initially investigated in patients with $\mathrm{BC}$ in a Phase I study. This study showed noteworthy anti-tumor activity, durable responses, and the favorable toxicity profile of atezolizumab. ${ }^{15,16}$ Based on these positive results, single-arm, two cohort, Phase II study (IMvigor 210) was launched. ${ }^{17,18}$ In one of the cohorts, patients with unresectable or metastatic UC whose disease had progressed after previous platinumbased chemotherapy were enrolled, showing durable antitumor activity and good tolerability; furthermore, there was an association between increased level of PD-L1 expression and increased responses. ${ }^{17}$ The PD-L1 expression was assessed by immunohistochemistry with VENTANA SP142 assay (Ventana Medical Systems, Inc, Tucson, AZ), and the PD-L1 expression on tumor-infiltrating immune cell (IC) status was defined by the percentage of PD-L1-positive immune cells in the tumor microenvironment: IC0 $(<1 \%)$, IC1 $(\geq 1 \%,<5 \%)$, and IC $2 / 3(\geq 5 \%)$.

To confirm the utility of atezolizumab in patients in a post-platinum-based chemotherapy setting, an open-label randomized Phase III trial (IMVigor 211) was launched, which compared atezolizumab with the investigator's choice of chemotherapy, including paclitaxel, docetaxel, and vinflunine. ${ }^{19}$ A total of 931 patients were randomly assigned in a $1: 1$ ratio to receive either atezolizumab $1200 \mathrm{mg}$ every 3 weeks $(n=467)$ or the investigator's choice of a chemotherapy regimen $(n=464)$. The primary endpoint of OS was assessed hierarchically in a population with high PD-L1 expression (IC2/3) followed by low PD-1 expression (IC1/2/3), and then by intention-to-treat populations. Unfortunately, in high PD-1-expressing population, OS did not differ significantly between the atezolizumab and chemotherapy arms, and the median OS was 11.1 and 10.6 months, respectively (HR 0.87; 95\% CI: $0.63-1.21 ; P=0.41)$. However, the safety profile for atezolizumab was favorable compared with chemotherapy, and exploratory analysis of the intention-to-treat population showed longer OS in the atezolizumab arm than the chemotherapy arm (HR 0.85; 95\% CI: 0.73-0.99). Taken together, these results suggest the benefit of atezolizumab in a post-platinum-based chemotherapy setting, despite the negative primary endpoint in the Phase III IMVigor 211 trial.

Nivolumab, an anti-PD-1 antibody, was investigated for its safety and anti-tumor activity in several solid tumors in an open-label, Phase I/II study (CheckMate 032). ${ }^{20,21}$ In patients with unresectable or metastatic UC whose disease progressed after previous platinum-based chemotherapy, nivolumab monotherapy was associated with a substantial and durable response, as well as acceptable safety. ${ }^{21}$ These results supported a single-arm Phase II study (CheckMate 275) in patients in post-platinumbased chemotherapy setting. ${ }^{22}$ A total of 270 patients were enrolled and treated with nivolumab $3 \mathrm{mg} / \mathrm{kg}$ every 2 weeks. Among 265 evaluable patients, ORR was approximately $20 \%$ with $2 \%$ CR. Based on this study, the FDA granted accelerated approval to nivolumab, and recommended the dose and schedule for the above indication as $240 \mathrm{mg}$ intravenously every 2 weeks.

Avelumab, an anti-PD-L1 antibody, was initially assessed in a Phase I, open-label, dose-expansion study in several solid malignancies (JAVELIN Solid Tumor study). ${ }^{23-25}$ To assess the safety profile in patients treated with avelumab and the anti-tumor activity of this drug in unresectable or metastatic UC, a pooled analysis of two cohorts from the JAVELIN Solid Tumor study was performed. ${ }^{26}$ A total of 249 patients were eligible and were treated with avelumab $10 \mathrm{mg} / \mathrm{kg}$ every 2 weeks. Among 161 evaluable patients, ORR was 17\% with $6 \% \mathrm{CR}$. Avelumab showed a manageable safety profile, and the most frequent AEs were infusion-related reactions (29\%) and fatigue (16\%). Based on these data, the FDA granted accelerated approval to avelumab and recommended a dose of $10 \mathrm{mg} / \mathrm{kg}$ intravenously over 60 mins every 2 weeks. To avoid infusion-related reactions, pre-medication with an antihistamine and acetaminophen prior to the first four infusions of avelumab is recommended. 
Durvalumab, an anti-PD-L1 antibody, was investigated for its safety and anti-tumor activity in a Phase I/II openlabel dose-escalation and dose-expansion study. ${ }^{27,28}$ Patients with unresectable or metastatic UC were enrolled and treated with durvalumab $10 \mathrm{mg} / \mathrm{kg}$ every 2 weeks. Among 182 evaluable patients with post-platinum-based chemotherapy, durvalumab demonstrated favorable antitumor activity, with an ORR of $18 \%$ and $3 \% \mathrm{CR}$, and a manageable safety profile. Based on these data, the FDA granted accelerated approval to durvalumab.

\section{First-line ICls for cisplatin-ineligible patients}

Up to $50 \%$ of patients with unresectable or metastatic UC who show impaired renal function are ineligible for cisplatin-based chemotherapy, which is the standard first-line treatment for these patients. ${ }^{29}$ Treatment alternatives including carboplatin-based combinations and single-drug chemotherapy are associated with inferior outcomes compared with cisplatin-based chemotherapy in cisplatineligible patients; therefore, the development of alternative treatment approaches in cisplatin-ineligible patients was anticipated. ${ }^{8,30,31}$ In 2017, the FDA granted accelerated approval to pembrolizumab and atezolizumab as first-line treatment for cisplatin-ineligible patients with unresectable or metastatic UC (Table 1).

\section{Pembrolizumab}

Pembrolizumab for cisplatin-ineligible patients was assessed in a single-arm Phase II study (KEYNOTE-052). ${ }^{14}$ Cisplatinineligible patients were defined as meeting at least one of the following criteria: Eastern Cooperative Oncology Group (ECOG) performance status 2, creatinine clearance $30-60 \mathrm{ml} / \mathrm{mins}$, grade $\geq 2$ audiometric hearing loss, grade $\geq 2$ peripheral neuropathy, or New York Heart Association Class III heart failure. A total of 370 patients received pembrolizumab $200 \mathrm{mg}$ every 3 weeks. With a median follow-up of 5 months, the ORR was $24 \%$ with $5 \% \mathrm{CR}$, and $83 \%$ of responses were ongoing at the time of cutoff. High PD-L1 expression, defined as a CPS of at least $10 \%$, was associated with high ORR of $38 \%$. Pembrolizumab showed acceptable tolerability in this study population. Based on the updated data of this study, the FDA granted accelerated approval as a first-line indication in cisplatin-ineligible patients with unresectable or metastatic UC. However, the FDA limited the use of pembrolizumab in these patients in June 2018, based on the data of an ongoing Phase III trial, KEYNOTE-
361 (ClinialTrials.gov Identifier: NCT02853305), in which patients were randomly assigned to receive pembrolizumab with or without chemotherapy, or chemotherapy alone. The Independent Data Monitoring Committee (DMC) found that patients with low PD-L1 expression, defined as CPS $<10 \%$, who received pembrolizumab alone had poorer survival compared with patients receiving platinum-based chemotherapy. Based on these results, the FDA revised the indications for pembrolizumab in cisplatin-ineligible patients. Pembrolizumab is now indicated for cisplatinineligible patients with high tumor PD-L1 expression (CPS at least $10 \%$ ), and patients who are ineligible for any platinum-containing chemotherapy regardless of tumor PD-L1 expression. ${ }^{32}$

\section{Atezolizumab}

Atezolizumab for cisplatin-ineligible patients was assessed in single-arm two-cohort Phase II study (IMvigor 210), as mentioned above. ${ }^{18}$ One of the cohorts consisted of previously untreated cisplatin-ineligible patients, and was treated with atezolizumab $1200 \mathrm{mg}$ every 3 weeks, in the same procedure as another cohort in a post-platinum-based chemotherapy setting. A total of 119 patients were enrolled and treated with atezolizumab, exhibiting an ORR of $23 \%$ and a CR of $9 \%$ with a median follow-up of 17.2 months. The FDA granted accelerated approval to atezolizumab as first-line indication in cisplatin-ineligible patients, but similarly to pembrolizumab, the FDA have limited the indication. Atezolizumab is now indicated for cisplatin-ineligible patients with high tumor PD-L1 expression (PD-L1 stained tumor-infiltrating immune cells covering $\geq 5 \%$ of the tumor area), and patients who are ineligible for any platinum-containing chemotherapy regardless of tumor PD-L1 expression.

\section{Adverse events associated with pembrolizumab}

The KEYNOTE-045 trial showed that treatment-related AEs of any grade were seen in $61 \%$ of patients treated with pembrolizumab, the most common AEs being pruritus $(20 \%)$, fatigue $(14 \%)$, and nausea $(11 \%) .{ }^{13}$ Severe AEs, including grade 3,4 , and 5 events, were observed in $15 \%$ of patients, while the incidence of each $\mathrm{AE}$ was less than 5\%. Wang et al reported the safety profile of pembrolizumab through a pooled analysis based on randomized controlled trials for solid cancers. ${ }^{33}$ They analyzed a total of 3,922 patients, and reported the incidence of all- 
grade AEs including rash (15\%), pain (14\%), pruritus $(18 \%)$, vitiligo (11\%), arthralgia (11\%), and dry mouth $(10.0 \%)$, while severe $\mathrm{AE}$ rates of rash, pruritus, and arthralgia were rare. The incidence rate of AEs is similar to those associated with other anti-PD-1/PD-L1 antibodies. $^{9}$

Immune-related AEs are characteristic events among ICIs, and physicians must closely monitor them to enable their management. The following immune-related AEs associated with pembrolizumab have been reported: pneumonitis, colitis, hepatitis, nephritis, hyper- or hypothyroidism, thyroiditis, hypophysitis, type 1 diabetes mellitus, arthritis, myositis, Guillain-Barré syndrome, pancreatitis, and skin reactions. ${ }^{34}$ In the KEYNOTE-045 trial, immunerelated AEs of any grade were recorded in $17 \%$ of patients, and the incidence of grade 3,4 , or 5 events included pneumonitis $(2 \%)$, colitis $(1 \%)$, and nephritis $(1 \%) .{ }^{13}$

\section{Ongoing Phase III clinical trials using pembrolizumab and other ICls}

Table 2 indicates the current ongoing Phase III trials of pembrolizumab in patients with unresectable or metastatic UC. Pembrolizumab monotherapy is the standard treatment with level 1 evidence for patients who failed first-line platinum-based chemotherapy. Therefore, it is necessary to design clinical trials with a control arm of pembrolizumab monotherapy for the development of new treatment in the post-platinum-based chemotherapy setting. Two Phase III trials of pembrolizumab in such patients are detailed in ClinicalTrials.gov.

Erdafitinib, a pan-fibroblast growth factor receptor (FGFR) tyrosine kinase inhibitor, showed clinical efficacy in patients with FGFR-altered unresectable or metastatic UC in a Phase II study, BLC2001, which was presented at the 2018 Genitourinary Cancers Symposium. The FDA have granted Breakthrough Therapy Designation for erdafitinib in the treatment of FGFR-mutated UC. A Phase III trial investigating erdafitinib monotherapy in the secondline setting was launched in patients with unresectable or metastatic UC and FGFR genetic aberrations. In this trial, erdafitinib was compared with pembrolizumab (control arm) in patients with prior chemotherapy (cohort 2), or with chemotherapy in patients with prior chemotherapy and ICI (cohort 1). The primary endpoint of this trial was OS. Meanwhile, the development of combination therapy of pembrolizumab with epacadostat had run into difficulties. Epacadostat is an inhibitor of indoleamine 2, 3-dioxygenase-1 (IDO1), which suppresses T-cell-mediated immune surveillance. ${ }^{35}$ A Phase I/II trial (ECHO-202/KEYNOTE037) showed that the combination of pembrolizumab and epacadostat was tolerated and that it exhibited encouraging antitumor activity in multiple advanced solid tumors. ${ }^{36}$ A Phase III trial (KEYNOTE-698/ECHO-303) was launched in patients who had failed first-line platinumbased chemotherapy. However, another Phase III study investigating the same combination therapy in patients with unresectable or metastatic melanoma failed to meet its primary endpoint of PFS, and thus the enrollment of patients for KEYNOTE-698/ECHO-303 was halted.

Currently, the standard first-line therapy in cisplatineligible patients with unresectable or metastatic UC is cisplatin-based chemotherapy. Pembrolizumab is being investigated as a first-line therapy with or without chemotherapy in platinum-eligible patients with unresectable or metastatic UC. A Phase III three-armed randomized trial is currently ongoing that includes pembrolizumab with the gemcitabine plus cisplatin or carboplatin arm, the pembrolizumab monotherapy arm, and the placebo with gemcitabine and cisplatin/carboplatin arm (KEYNOTE-361). The total estimated enrollment is 990 patients, and the co-primary endpoints of this trial are PFS and OS. Atezolizumab is also being investigated in a Phase III trial with a similar design (IMvigor130) (ClinicalTrials.gov Identifier: NCT02807636).

Another strategy of combination therapy is to use antiPD-1/PD-L1 antibodies with other ICIs such as those targeting the CTLA-4 pathway, including ipilimumab and tremelimumab. A Phase III trial evaluating nivolumab $1 \mathrm{mg} / \mathrm{kg}$ and ipilimumab $3 \mathrm{mg} / \mathrm{kg}$ combination with or without platinum-based chemotherapy in the first-line setting (CheckMate 901) (ClinicalTrials.gov Identifier: NCT03036098) is ongoing. Durvalumab monotherapy or combination therapy of durvalumab with tremelimumab are also being compared with platinum-based chemotherapy in an open-label Phase III trial (DANUBE trial) (ClinicalTrials.gov Identifier: NCT02261220). In addition to first- and second-line therapy for unresectable or metastatic UC, the role of avelumab in maintenance therapy is being evaluated in patients with advanced UC who have completed at least four cycles of platinum-based chemotherapy without evidence of disease progression (JAVELIN Bladder 100 study) (ClinicalTrials.gov Identifier: NCT02603432). 


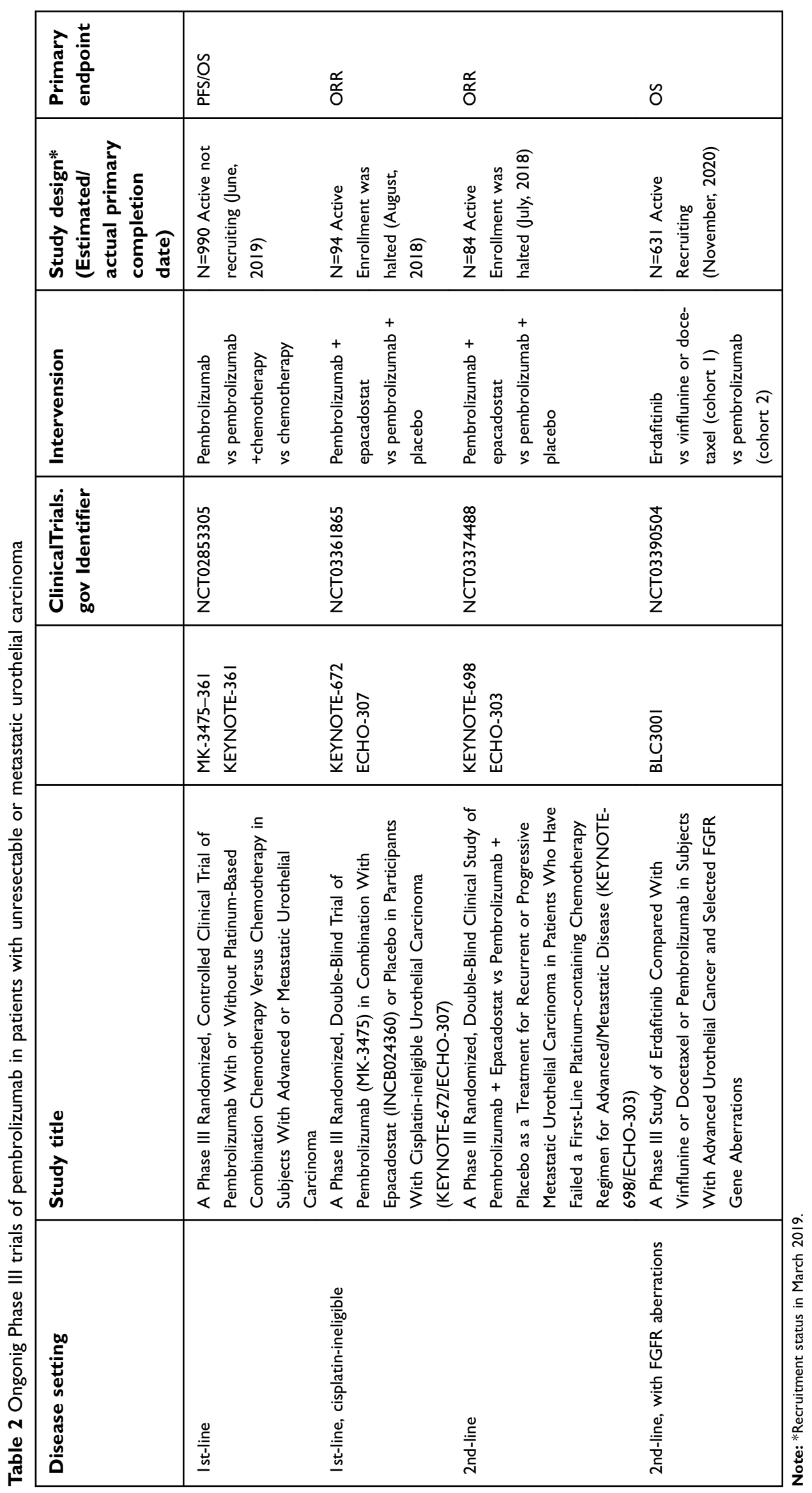


The results of these trials of ICIs might influence treatment strategies in second-line or following setting of unresectable or metastatic UC patients. Recently, the FDA has granted Breakthrough Therapy Designations for enfortumab vedotin and erdafitinib for patients following platinum-based chemotherapy. ${ }^{37}$ Several Phase III trials are ongoing to evaluate the efficacy of enfortumab vedotin and FGFR inhibitors including erdafitinib, in post-ICIs setting for unresectable or metastatic UC patients.

\section{Conclusions and future view}

Pembrolizumab is a potential first-choice second-line therapy for unresectable or metastatic UC patients following platinum-based chemotherapy, because it is the only biologic to have strong evidence of efficacy in this setting. Several Phase III trials are ongoing to evaluate the efficacy and toxicity of ICIs with chemotherapy combination therapies, and ICIs with other ICIs with or without chemotherapy as first-line therapy. The results of these trials might influence the treatment strategies for unresectable or metastatic UC patients.

Another notable point is that the treatment using ICIs is currently investigating in non-metastatic patients. Multiple trials are ongoing which investigate ICI mono- or combination-therapy in muscle-invasive resectable $\mathrm{BC}$, as neoadjuvant or adjuvant treatment, and also in non-muscle-invasive BC. In the near future, ICIs might be incorporated into the standard of care for these non-advanced disease, and it will be strongly required to develop the novel treatment in patients with post-ICI setting.

In addition, it is necessary to consider the costeffectiveness of ICI-containing therapies because ICIs are priced high. Although considerable patients have longterm durable response, some patients do not benefit from these treatments. Therefore, the identification of patients who benefit or do not benefit from these treatments is the key for personalized medicine, which might improve the cost-effectiveness. Unfortunately, the current biomarker, PD-L1 staining, showed inconsistence results in the trials using ICIs, and is far from decision-making tool. Further research to find biomarkers for identifying potential treatment responders is required.

\section{Abbreviations}

$\mathrm{AE}$, adverse event; $\mathrm{BC}$, bladder cancer; $\mathrm{CR}$, complete response; CTLA-4, cytotoxic T lymphocyte-associated protein 4; FDA, the US Food and Drug Administration; FGFR, fibroblast growth factor receptor; ICI, immune checkpoint inhibitor; ORR, overall response rate; OS, overall survival; PFS, progression-free survival; PD-1, programmed cell death 1; PD-L1, programmed death ligand 1; UC, urothelial carcinoma.

\section{Acknowledgments}

This study was supported in part by JSPS KAKENHI Grant Number JP16K11010. The authors would like to thank H. Nikki March, PhD, from Edanz Group (www.edanzediting.com/ac) for editing a draft of this manuscript.

\section{Disclosure}

ME reports honoraria for speaking from Ono, BMS, MSD, Chugai, Pfizer, AstraZeneca, Astellas, and Bayer. He also reports personal fees from MSD, outside the submitted work. The authors report no other conflicts of interest in this work.

\section{References}

1. Ferlay J, Steliarova-Foucher E, Lortet-Tieulent J, et al. Cancer incidence and mortality patterns in Europe: estimates for 40 countries in 2012. Eur J Cancer. 2013;49(6):1374-1403.

2. Burger M, Catto JW, Dalbagni G, et al. Epidemiology and risk factors of urothelial bladder cancer. Eur Urol. 2013;63(2):234-241. doi:10.1016/j.eururo.2012.07.033

3. Advanced Bladder Cancer Meta-analysis C. Neoadjuvant chemotherapy in invasive bladder cancer: update of a systematic review and meta-analysis of individual patient data advanced bladder cancer (ABC) meta-analysis collaboration. Eur Urol. 2005;48(2):202-205. discussion 205-206. doi:10.1016/j.eururo.2005.04.006

4. Rosenberg JE, Carroll PR, Small EJ. Update on chemotherapy for advanced bladder cancer. $J$ Urol. 2005;174(1):14-20. doi:10.1097/01. ju.0000162039.38023.5f

5. Sternberg CN, Yagoda A, Scher HI, et al. M-VAC (methotrexate, vinblastine, doxorubicin and cisplatin) for advanced transitional cell carcinoma of the urothelium. $J$ Urol. 1988;139(3):461-469.

6. Sternberg CN, de Mulder PH, Schornagel JH, et al. Randomized phase III trial of high-dose-intensity methotrexate, vinblastine, doxorubicin, and cisplatin (MVAC) chemotherapy and recombinant human granulocyte colony-stimulating factor versus classic MVAC in advanced urothelial tract tumors: European organization for research and treatment of cancer protocol no. 30924. J Clin Oncol. 2001;19 (10):2638-2646. doi:10.1200/JCO.2001.19.10.2638

7. Sternberg CN, de Mulder P, Schornagel JH, et al. Seven year update of an EORTC phase III trial of high-dose intensity M-VAC chemotherapy and G-CSF versus classic M-VAC in advanced urothelial tract tumours. Eur J Cancer. 2006;42(1):50-54. doi:10.1016/j.ejca.2005.08.032

8. von der Maase H, Sengelov L, Roberts JT, et al. Long-term survival results of a randomized trial comparing gemcitabine plus cisplatin, with methotrexate, vinblastine, doxorubicin, plus cisplatin in patients with bladder cancer. J Clin Oncol. 2005;23(21):4602-4608. doi:10.1200/ JCO.2005.07.757

9. Rouanne M, Roumiguie M, Houede N, et al. Development of immunotherapy in bladder cancer: present and future on targeting $\mathrm{PD}(\mathrm{L}) 1$ and CTLA-4 pathways. World J Urol. 2018;36(11):1727-1740. doi:10.1007/s00345-018-2332-5 
10. Schreiber RD, Old LJ, Smyth MJ. Cancer immunoediting: integrating immunity's roles in cancer suppression and promotion. Science. 2011;331(6024):1565-1570. doi:10.1126/science.1203486

11. Dequen P, Lorigan P, Jansen JP, van Baardewijk M, Ouwens MJ, Kotapati S. Systematic review and network meta-analysis of overall survival comparing $3 \mathrm{mg} / \mathrm{kg}$ ipilimumab with alternative therapies in the management of pretreated patients with unresectable stage III or IV melanoma. Oncologist. 2012;17(11):1376-1385. doi:10.1634/ theoncologist.2011-0427

12. Plimack ER, Bellmunt J, Gupta S, et al. Safety and activity of pembrolizumab in patients with locally advanced or metastatic urothelial cancer (KEYNOTE-012): a non-randomised, open-label, phase 1b study. Lancet Oncol. 2017;18(2):212-220. doi:10.1016/ S1470-2045(17)30007-4

13. Bellmunt $J$, de Wit R, Vaughn DJ, et al. Pembrolizumab as second-line therapy for advanced urothelial carcinoma. $N$ Engl J Med. 2017;376(11):1015-1026. doi:10.1056/NEJMoa1613683

14. Balar AV, Castellano D, O`Donnell PH, et al. First-line pembrolizumab in cisplatin-ineligible patients with locally advanced and unresectable or metastatic urothelial cancer (KEYNOTE-052): a multicentre, single-arm, phase 2 study. Lancet Oncol. 2017;18 (11):1483-1492. doi:10.1016/S1470-2045(17)30616-2

15. Powles T, Eder JP, Fine GD, et al. MPDL3280A (anti-PD-L1) treatment leads to clinical activity in metastatic bladder cancer. Nature 2014;515(7528):558-562. doi:10.1038/nature13904

16. Petrylak DP, Powles T, Bellmunt J, et al. Atezolizumab (MPDL3280A) monotherapy for patients with metastatic urothelial cancer: long-term outcomes from a phase 1 study. JAMA Oncol. 2018;4(4):537-544. doi:10.1001/jamaoncol.2017.5440

17. Rosenberg JE, Hoffman-Censits J, Powles T, et al. Atezolizumab in patients with locally advanced and metastatic urothelial carcinoma who have progressed following treatment with platinum-based chemotherapy: a single-arm, multicentre, phase 2 trial. Lancet. 2016;387 (10031):1909-1920. doi:10.1016/S0140-6736(16)00561-4

18. Balar AV, Galsky MD, Rosenberg JE, et al. Atezolizumab as first-line treatment in cisplatin-ineligible patients with locally advanced and metastatic urothelial carcinoma: a single-arm, multicentre, phase 2 trial. Lancet. 2017;389(10064):67-76. doi:10.1016/S0140-6736(16)32455-2

19. Powles T, Duran I, van der Heijden MS, et al. Atezolizumab versus chemotherapy in patients with platinum-treated locally advanced or metastatic urothelial carcinoma (IMvigor211): a multicentre, open-label, phase 3 randomised controlled trial. Lancet. 2018;391 (10122):748-757. doi:10.1016/S0140-6736(17)33297-X

20. Antonia SJ, Lopez-Martin JA, Bendell J, et al. Nivolumab alone and nivolumab plus ipilimumab in recurrent small-cell lung cancer (CheckMate 032): a multicentre, open-label, phase 1/2 trial. Lancet Oncol. 2016;17(7):883-895. doi:10.1016/S1470-2045(16)30098-5

21. Sharma P, Callahan MK, Bono $P$, et al. Nivolumab monotherapy in recurrent metastatic urothelial carcinoma (CheckMate 032): a multicentre, open-label, two-stage, multi-arm, phase $1 / 2$ trial. Lancet Oncol. 2016;17(11):1590-1598. doi:10.1016/S1470-2045(16)30496-X

22. Sharma P, Retz M, Siefker-Radtke A, et al. Nivolumab in metastatic urothelial carcinoma after platinum therapy (CheckMate 275): a multicentre, single-arm, phase 2 trial. Lancet Oncol. 2017;18 (3):312-322. doi:10.1016/S1470-2045(17)30065-7

23. Heery CR, O'Sullivan-Coyne G, Madan RA, et al. Avelumab for metastatic or locally advanced previously treated solid tumours (JAVELIN solid tumor): a phase 1a, multicohort, dose-escalation trial. Lancet Oncol. 2017;18(5):587-598. doi:10.1016/S14702045(17)30239-5
24. Gulley JL, Rajan A, Spigel DR, et al. Avelumab for patients with previously treated metastatic or recurrent non-small-cell lung cancer (JAVELIN solid tumor): dose-expansion cohort of a multicentre, open-label, phase 1b trial. Lancet Oncol. 2017;18(5):599-610. doi:10.1016/S1470-2045(17)30240-1

25. Apolo AB, Infante JR, Balmanoukian A, et al. Avelumab, an anti-programmed death-ligand 1 antibody, in patients with refractory metastatic urothelial carcinoma: results from a multicenter, phase Ib study. $J$ Clin Oncol. 2017;35(19):2117-2124. doi:10.1200/JCO.2016.71.6795

26. Patel MR, Ellerton J, Infante JR, et al. Avelumab in metastatic urothelial carcinoma after platinum failure (JAVELIN solid tumor): pooled results from two expansion cohorts of an open-label, phase 1 trial. Lancet Oncol. 2018;19(1):51-64. doi:10.1016/S1470-2045(17) 30900-2

27. Massard C, Gordon MS, Sharma S, et al. Safety and efficacy of durvalumab (MEDI4736), an anti-programmed cell death ligand-1 immune checkpoint inhibitor, in patients with advanced urothelial bladder cancer. J Clin Oncol. 2016;34(26):3119-3125. doi:10.1200/ JCO.2016.67.9761

28. Powles T, O'Donnell PH, Massard C, et al. Efficacy and safety of durvalumab in locally advanced or metastatic urothelial carcinoma: updated results from a phase 1/2 open-label study. JAMA Oncol. 2017;3(9):e172411. doi:10.1001/jamaoncol.2017.2411

29. Galsky MD, Hahn NM, Rosenberg J, et al. Treatment of patients with metastatic urothelial cancer "unfit" for Cisplatin-based chemotherapy. J Clin Oncol. 2011;29(17):2432-2438. doi:10.1200/JCO.2011.34.8433

30. Galsky MD, Chen GJ, Oh WK, et al. Comparative effectiveness of cisplatin-based and carboplatin-based chemotherapy for treatment of advanced urothelial carcinoma. Ann Oncol. 2012;23(2):406-410. doi:10.1093/annonc/mdr156

31. De Santis M, Bellmunt J, Mead G, et al. Randomized phase II/III trial assessing gemcitabine/carboplatin and methotrexate/carboplatin/vinblastine in patients with advanced urothelial cancer who are unfit for cisplatin-based chemotherapy: EORTC study 30986. J Clin Oncol. 2012;30(2):191-199. doi:10.1200/JCO.2011.37.3571

32. Suzman DL, Agrawal S, Ning YM, et al. FDA approval summary: atezolizumab or pembrolizumab for the treatment of patients with advanced urothelial carcinoma ineligible for cisplatin-containing chemotherapy. Oncologist. 2019;24(4):563-569. doi: 10.1634/theoncologist.2018-0084.

33. Wang M, Ma X, Guo L, Xia F. Safety and efficacy profile of pembrolizumab in solid cancer: pooled reanalysis based on randomized controlled trials. Drug Des Devel Ther. 2017;11:2851-2860. doi:10.2147/DDDT.S146286

34. Sundahl N, Rottey S, De Maeseneer D, Ost P. Pembrolizumab for the treatment of bladder cancer. Expert Rev Anticancer Ther. 2018;18 (2):107-114. doi:10.1080/14737140.2018.1421461

35. Liu X, Shin N, Koblish HK, et al. Selective inhibition of IDO1 effectively regulates mediators of antitumor immunity. Blood. 2010;115(17):3520-3530. doi:10.1182/blood-2009-09-246124

36. Mitchell TC, Hamid O, Smith DC, et al. Epacadostat plus pembrolizumab in patients with advanced solid tumors: phase I results from a multicenter, open-label phase I/II trial (ECHO-202/KEYNOTE037). J Clin Oncol. 2018; doi:10.1200/JCO.2018.78.9602. [Epub ahead of print]

37. Hanna KS. Updates and novel treatments in urothelial carcinoma. J Oncol Pharm Pract. 2019;25(3):648-656. doi:10.1177/1078155218805141 


\section{Publish your work in this journal}

Cancer Management and Research is an international, peer-reviewed The manuscript management system is completely online and includes open access journal focusing on cancer research and the optimal use of a very quick and fair peer-review system, which is all easy to use. preventative and integrated treatment interventions to achieve improved Visit http://www.dovepress.com/testimonials.php to read real quotes outcomes, enhanced survival and quality of life for the cancer patient.

from published authors. 